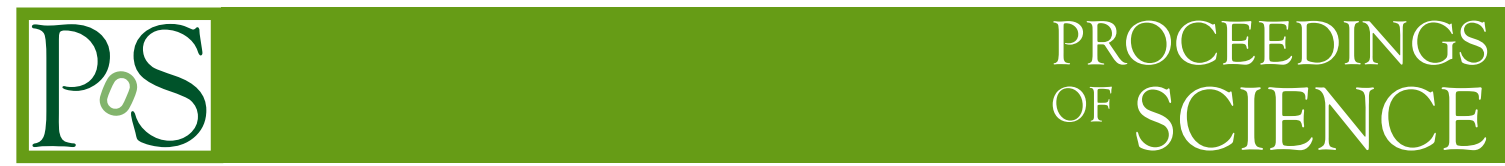

\title{
Search for exotic heavy quark partners
}

\section{Davide Pagano* for the CMS Collaboration}

Université catholique de Louvain

E-mail: davide.pagano@cern.ch

A summary of the CMS Collaboration results on searches for new heavy quarks, predicted by many models beyond the Standard Model (BSM), is presented. Results were produced with data collected at the Large Hadron Collider at a center-of-mass energy of $7 \mathrm{TeV}$ and $8 \mathrm{TeV}$, with integrated luminosities ranging between 5.0 and $19.6 \mathrm{fb}^{-1}$. No evidence for new massive quarks was observed and exclusion limits were set for some BSM scenarios.

XXI International Workshop on Deep-Inelastic Scattering and Related Subject -DIS2013, 22-26 April 2013

Marseilles, France

${ }^{*}$ Speaker. 


\section{Introduction}

At present, there is experimental evidence for just three generations of fermions in the Standard Model (SM), though their number is not fixed by the theory. The only theoretical constraint comes from the QCD asymptotic freedom, which requires the number of generations to be less than 9 [1]. On the the other hand, the existence of a fourth generation of fermions is subject to some experimental constraints from precision electroweak data [2] and direct searches performed at the Tevatron and at the Large Hadron Collider (LHC).

Searches for new heavy quarks are of particular interest, as these quarks are predicted in many new physics models aimed at solving some of the limitations of the SM. The most natural extension of the SM consists of adding a fourth chiral generation of fermions, which, providing additional sources of $\mathrm{CP}$ violation, could explain the observed matter-antimatter asymmetry in the universe [1]. Another possibility is the addition of new heavy vector-like quarks, defined as quarks for which both chiralities have the same transformation properties under the SM gauge group. Vector-like quarks are predicted by many extensions of the SM, like little Higgs models or extra dimension [3].

Recent results of Higgs searches at LHC have additionally constrained the existence of new heavy quarks. In particular, a fourth chiral generation of quarks is disfavored by the observation of the SM-like Higgs boson with mass of $126 \mathrm{GeV}$, although it could still be incorporated in beyond SM scenarios with an extended Higgs sector [4].

This paper presents the results of the CMS direct searches for new heavy quarks, based on data collected in 2011 and 2012 at a center-of-mass energy of $7 \mathrm{TeV}$ and $8 \mathrm{TeV}$, with integrated luminosities ranging between 5.0 and $19.6 \mathrm{fb}^{-1}$. A detailed description of the CMS detector is provided in Ref. [5], while the full descriptions of the analyses presented here can be found in Ref. $[6,7,8,9]$.

\section{Search for new heavy quarks decaying to $t V$}

This section presents the CMS search for pair-produced heavy quarks $Q$, decaying exclusively to a $t$-quark and a $W$ or $Z$ boson [6]. The analysis was performed using $5 \mathrm{fb}^{-1}$ of data at $\sqrt{\mathrm{s}}=7$ TeV. For $Q \rightarrow t W$, the $Q \bar{Q}$ final state contains four $W$ bosons and two $b$-quarks, while, for $Q \rightarrow t Z$, it contains two $Z$ bosons, two $W$ bosons and two $b$-quarks. In both cases, the search was performed with events where one of the $W$ bosons decayed leptonically, while the other bosons decayed to jet pairs.

Events were requested to have exactly one muon (electron), with transverse momentum $p_{T}>$ 42 (35) $\mathrm{GeV}$, at least four jets with $p_{T}>100,60,50,35 \mathrm{GeV}$ and transverse missing energy $E_{T}^{\text {miss }}$ $\geq 20 \mathrm{GeV}$. They were then split into four exclusive categories according to the jet multiplicity $\left(N_{j}\right): N_{j}=4,5,6$, and $\geq 7$ jets. The search for the $Q \bar{Q}$ signal was performed by fitting the data $S_{T}$ distribution as a function of the jet multiplicity, where $S_{T}$ was defined as the scalar sum of the lepton and jets transverse momenta and the $E_{T}^{\text {miss }}$. Figure 1 left (right) shows the observed and expected 95\% CL upper limits on the signal production cross-section, as a function of the $Q$ quark mass $\left(M_{Q}\right)$, for $Q$ decaying exclusively to $t W(t Z)$. Assuming a strong production mechanism for the signal model, masses below 675 (625) GeV were excluded for $Q \rightarrow t W(Q \rightarrow t Z)$. 

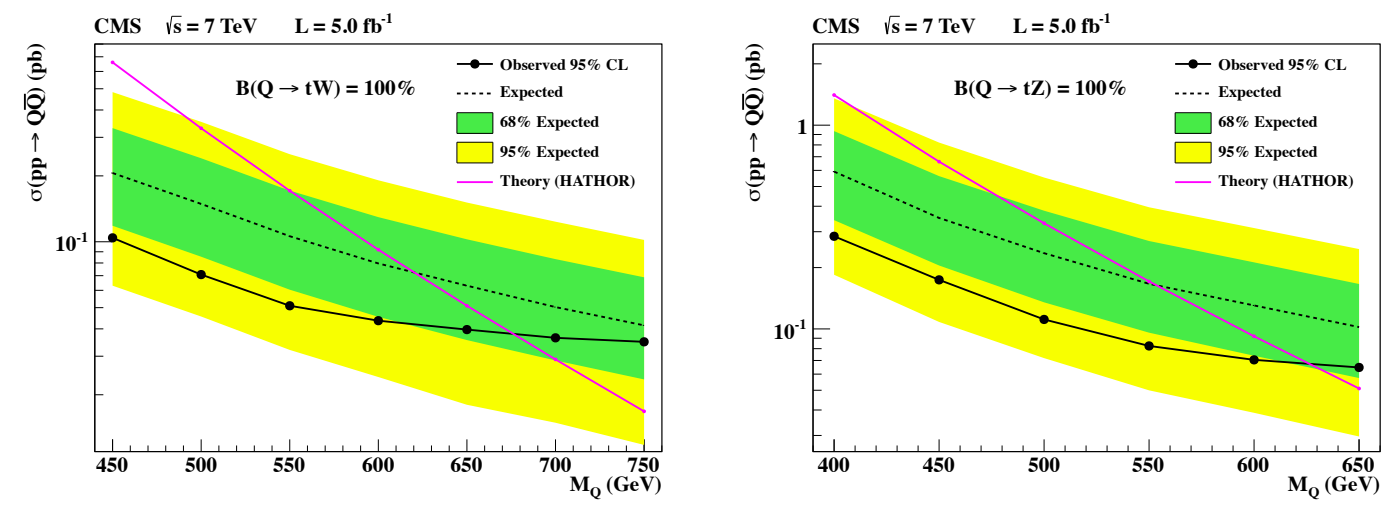

Figure 1: $Q \rightarrow t V$ search - Expected and observed 95\% CL upper limits on the signal cross-section as a function of the $Q$ quark mass, assuming $B(Q \rightarrow t W)=100 \%$ (left) and $B(Q \rightarrow t Z)=100 \%$ (right) [6].

\section{Search for new heavy top partners with charge $5 \mathrm{e} / 3$}

In this section a summary of the CMS search for pair-produced heavy top partners with charge $5 \mathrm{e} / 3\left(T_{5 / 3}\right)$, using $19.6 \mathrm{fb}^{-1}$ of data at $\sqrt{s}=8 \mathrm{TeV}$, is given [7]. A branching fraction of $100 \%$ for the decay $T_{5 / 3} \rightarrow t W$ was assumed, so that the considered signal final state contained two pairs of opposite-charge $W$ bosons and two $b$-quarks. The search was performed with events where two same-sign $W$ bosons decayed leptonically, while the other bosons decayed to jet pairs. For high values of the $T_{5 / 3}$ quark mass, the $t$-quarks and $W$ bosons can be highly boosted and, consequently, jets from their hadronic decays could merge into one wide jet. For these cases, the analysis benefited from the use of dedicated reconstruction techniques, based on the Cambridge-Aachen (CA) clustering algorithm [10], which looked for substructures within wide jets and use them to identify boosted top quarks and $W$ bosons [11] [12].

Events were requested to have exactly two isolated same-sign leptons $(\mu \mu, e e$ or $e \mu)$ with $p_{T}>$ $30 \mathrm{GeV}$ and at least five constituents, where CA jets, identified to be originated from hadronically decays of boosted $W$ bosons and $t$-quarks, counted as two and three constituents respectively. Events were also required to have $H_{T}>900 \mathrm{GeV}$, with $H_{T}$ defined as the scalar sum of the leptons and jets $p_{T}$. A counting experiment, using the total number of events in the three channels, was performed. Figure 2 shows the expected and observed limits on the signal production cross-section, as well as the theoretical prediction [13]: $T_{5 / 3}$ quarks with masses below $770 \mathrm{GeV}$ were excluded.

\section{Inclusive search for a sequential fourth generation of quarks}

This section summarizes the inclusive CMS search for heavy $b$-quark and $t$-quark partners $\left(b^{\prime}\right.$ and $t^{\prime}$ ), using $5.0 \mathrm{fb}^{-1}$ of data at $\sqrt{s}=7 \mathrm{TeV}$ [8]. In presence of a $4^{\text {th }}$ generation of quarks, an extension of the Cabibbo-Kobayashi-Maskawa (CKM) matrix is required. For simplicity, a model with only one free parameter $A$ and degenerate masses for $t^{\prime}$ and $b^{\prime}\left(m_{t^{\prime}}=m_{b^{\prime}}\right)$ was assumed in the search. As a result, the $4 \times 4$ CKM matrix was rewritten as in Eq. 4.1 , where $\sqrt{A}=\left|V_{t b}\right|$. 


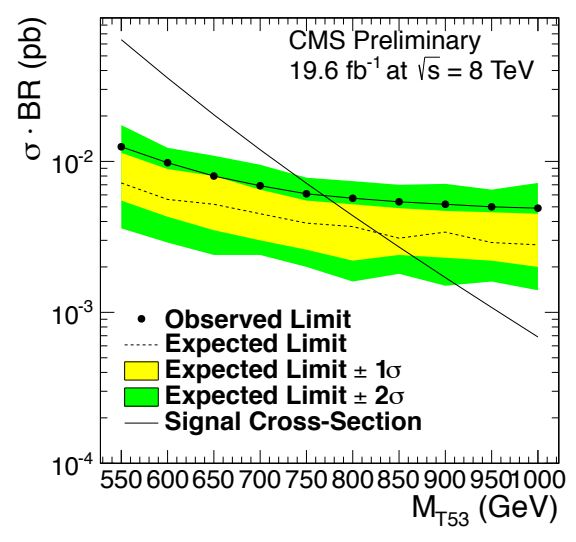

Figure 2: $T_{5 / 3}$ search - Expected and observed 95\% C.L. limits on the signal cross-section times branching ratio as a function of $M_{T 5 / 3}$, [7].

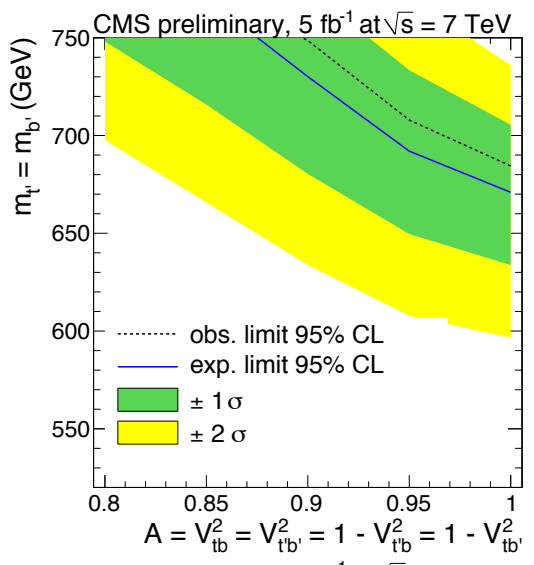

Figure 3: inclusive $t^{\prime}$ and $b^{\prime}$ search - 95\% CL exclusion limit on $m_{t^{\prime}}=m_{b^{\prime}}$ as a function of the $V_{C K M}^{4 \times 4}$ parameter $A[8]$.

$$
V_{C K M}^{4 x 4}=\left(\begin{array}{cccc}
V_{u d} & V_{u s} & V_{u b} & V_{u b^{\prime}} \\
V_{c d} & V_{c s} & V_{c b} & V_{c b^{\prime}} \\
V_{t d} & V_{t s} & V_{t b} & V_{t b^{\prime}} \\
V_{t^{\prime} d} & V_{t^{\prime} s} & V_{t^{\prime} b} & V_{t^{\prime} b^{\prime}}
\end{array}\right)=\left(\begin{array}{cccc}
1 & 0 & 0 & 0 \\
0 & 1 & 0 & 0 \\
0 & 0 & \sqrt{A} & \sqrt{1-A} \\
0 & 0 & -\sqrt{1-A} & \sqrt{A}
\end{array}\right)
$$

Single $\left(t^{\prime} b\right.$ and $\left.b^{\prime} t\right)$ and pair $\left(t^{\prime} \bar{t}^{\prime}, t^{\prime} b^{\prime}\right.$ and $\left.b^{\prime} \bar{b}^{\prime}\right)$ production processes for $b^{\prime}$ and $t^{\prime}$ quarks were considered. Under the analysis assumptions, the branching fractions of $t^{\prime} \rightarrow b W$ and $b^{\prime} \rightarrow t W \rightarrow$ $b W W$ were almost equal to $100 \%$, so that the considered final states contained two $b$-quarks and one to four $W$ bosons, depending on the production mechanism. Three different channels were defined: the single lepton channel, the same-sign dilepton channel and the three leptons channel.

For the single lepton channel, events were requested to have exactly one isolated lepton ( $\mu$ or $e)$ with $p_{T}>40$ and $E_{T}^{\text {miss }}>40 \mathrm{GeV}$. They were then classified according to the number of $b$-jets and hadronically decaying $W$ boson candidates. The $S_{T}$ distribution (defined in Section 2) and the reconstructed mass of the hadronic $b W$ system were used to look for the signal. For the dilepton (trilepton) channel, events were requested to have two same-sign leptons (three leptons) with $p_{T}$ $>20 \mathrm{GeV}$, at least four (two) $b$-jets and $E_{T}^{\text {miss }}>40 \mathrm{GeV}$. A counting experiment was performed in both the dilepton and trilepton channels. Figure 3 shows the expected and observed 95\% CL exclusion limits on $m_{t^{\prime}}=m_{b^{\prime}}$, as a function of $A$, for the combination of the three channels. For $A=1$, the observed (expected) limit was found to be 683 (670) GeV.

\section{Search for $b^{\prime}$ quarks with three or more leptons and b-tags}

This section summarizes the inclusive CMS search for anomalous production of events with three or more leptons, focusing on the interpretation of the results in the context of heavy $b^{\prime}$ quarks decaying to $t W$ or $b Z$ [9]. The analysis was performed using $9.2 \mathrm{fb}^{-1}$ of data at $\sqrt{s}=8 \mathrm{TeV}$.

Events were requested to have at least three isolated leptons with $p_{T}>10 \mathrm{GeV}(20 \mathrm{GeV}$ for the leading), including at most one hadronic $\tau$. They were then classified according to several quan- 

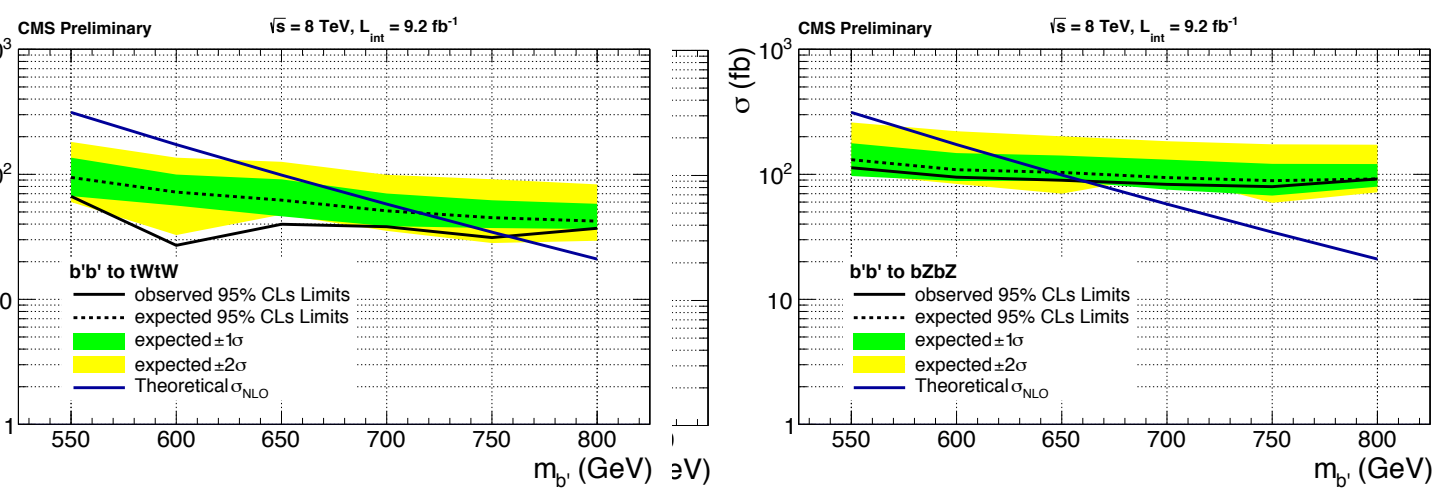

Figure 4: $b^{\prime} \rightarrow t W(t Z)$ search - Expected and observed 95\% C.L. limits on the signal production crosssection, as a function of the $b^{\prime}$ mass, for $b^{\prime}$ decaying exclusively to $t W$ (left) and $b Z$ (right) [9].

tities: the number of opposite-sign same-flavor (OSSF) lepton pairs; the number of reconstructed hadronic $\tau$ and $b$-jets; the event $S_{T}$; the number of OSSF pairs with dilepton mass in the $Z$ mass window ([75 GeV, $105 \mathrm{GeV}])$. This classification resulted in 90 (150) channels with 3 (4) leptons.

Channels with four (three) leptons, one (two) OSSF, no hadronic $\tau$, at least one $b$-jets and no $(\geq 1)$ OSSF pairs compatible with the $Z$ boson were used to search for pair-produced $b^{\prime}$, decaying to $t W(b Z)$. Expected and observed yields in the considered signal regions were combined to set exclusion limits. Figure 4 left (right) shows the 95\% CL upper limits on the signal production cross-section, as a function of the $b^{\prime}$ quark mass $\left(m_{b^{\prime}}\right)$, for $b^{\prime}$ decaying exclusively to $t W(b Z)$. The exclusion limit on $m_{b^{\prime}}$ was found to be 760 (660) GeV for $b^{\prime} \rightarrow t W\left(b^{\prime} \rightarrow b Z\right)$.

\section{Conclusions}

A summary of CMS searches for new heavy quarks has been presented. Results were produced using $p-p$ collision data at $\sqrt{s}=7 \mathrm{TeV}$ and $8 \mathrm{TeV}$, with integrated luminosities ranging between 5.0 and $19.6 \mathrm{fb}^{-1}$. A fourth generation of heavy quarks was searched for considering different production mechanisms and final states, with no evidence of its existence.

\section{References}

[1] B. Holdom, W. S. Hou, T. Hurth, M. L. Mangano, S. Sultansoy and G. Unel, PMC Phys. A 3, 4 (2009)

[2] J. Erler and P. Langacker, hep-ph/0407097.

[3] S. Kanemura, Y. Okada, E. Senaha and C. -P. Yuan, Phys. Rev. D 70, 115002 (2004)

[4] A. Djouadi and A. Lenz, Phys. Lett. B 715, 310 (2012) [arXiv:1204.1252 [hep-ph]].

[5] S. Chatrchyan et al. [CMS Collaboration], JINST 3, S08004 (2008).

[6] S. Chatrchyan et al. [CMS Collaboration], JHEP 01 (2013) 154

[7] [CMS Collaboration], CMS-PAS-B2G-12-012 (2013).

[8] [CMS Collaboration], CMS-PAS-EXO-11-098 (2012). 
[9] [CMS Collaboration], CMS-PAS-SUS-12-027 (2013).

[10] Y. L. Dokshitzer, G. D. Leder, S. Moretti and B. R. Webber, JHEP 9708, 001 (1997)

[11] D. E. Kaplan, K. Rehermann, M. D. Schwartz and B. Tweedie, Phys. Rev. Lett. 101, 142001 (2008)

[12] S. D. Ellis, C. K. Vermilion and J. R. Walsh, Phys. Rev. D 80, 051501 (2009)

[13] J. Mrazek and A. Wulzer, Phys. Rev. D 81, 075006 (2010) 